\title{
Correction of Foot Deformities from Charcot Arthropathy with the Taylor Spatial Frame: A 7-14-year Follow-up
}

\author{
Om Lahoti ${ }^{1}$, Naveen Abhishetty ${ }^{2}$, Sandesh Shetty ${ }^{3}$
}

\begin{abstract}
Charcot arthropathy related foot and ankle deformities are a serious challenge. Surgical treatment of these deformities is now well established. Conventional surgical treatment includes extensive surgical exposure, excision of bone, acute correction and internal fixation, which is not always appropriate in presence of active ulceration, infection and poor bone quality. A minimally invasive approach to osteotomies and gradual correction of deformities using a circular frame are proving helpful in minimizing the complications. Taylor Spatial Frame (TSF) hexapod with its various modules is well suited for a range of foot and ankle deformities. We have advocated minimally invasive targeted hind and mid foot osteotomies and gradual correction with Taylor Spatial Frame (TSF) in 10 patients with recurrent ulceration and deformity. There are 2 female and 8 male patients in this cohort. Appropriate TSF module was chosen for each patient- a long bone module for ankle and hindfoot deformities ( 4 patients) and a forefoot $6 \times 6$ butt frame ( 6 patients) for foot deformities. An osteotomy through the midfoot was performed in all chronic stable foot deformity cases. In the ankle and hindfoot deformities, a combination of soft tissue distraction correction of equinus and acute correction of hindfoot deformity through a calcaneal osteotomy, were used. Our outcome measures are complete healing of the ulcers and resolution of infection, clinically plantigrade foot and ability to wear regular or diabetic footwear. Complications included eight episodes of pin infection that responded to oral antibiotics only and two pin breakages. We achieved ulcer and infection free plantigrade feet that fit in to regular or diabetic footwear in 9 out of 10 patients. 9 patients remain ulcer and infection free at a minimum of 7 years and maximum of 14 years follow up. Taylor Spatial Frame treatment provides an alternative to conventional surgery in high-risk complex Charcot neuroarthropathy foot and ankle deformities.

Keywords: Charcot neuroarthropathy, Circular frame, Cohort study, Diabetic foot ulcers, Forefoot butt $6 \times 6$, Long bone TSF module, Rockerbottom foot deformity, Taylor spatial frame.
\end{abstract}

Strategies in Trauma and Limb Reconstruction (2021): 10.5005/jp-journals-10080-1525

\section{INTRODUCTION}

Foot problems related to diabetes are a serious surgical challenge. In the United Kingdom, there are an estimated 4.5 million people with diabetes and around $10 \%$ are likely to develop a foot ulcer in their lifetime. ' With a 5-year mortality rate of 50\% in those with ulcers and $80 \%$ amongst diabetes-related amputees, it is stated that diabetic foot ulcer mortality rates are similar or even worse than many types of common cancers. ${ }^{2}$ Well-established foot care pathways have shown a reduction in the first presentation of diabetic foot ulceration but failed to reduce recurrent ulceration. ${ }^{3}$ Charcot neuroarthropathy is progressive, and microtrauma potentiates it, resulting in the steady destruction of bone, subluxation of joints and a resultant deformity. Thus, in the acute phase, immobilisation and unloading of the foot are essential to prevent deformities from developing. However, orthotics and braces do not always support unstable joints fully and patients may develop a range of foot deformities which promote ulceration. ${ }^{4}$ The traditional surgical approach is to excise wedges or segments of bone through large incisions and often through compromised skin. Internal fixation is almost always used after such procedures which further increases the risk of wound breakdown and infection. ${ }^{5,6}$ Metal implant failure is also common. Circular external fixators have several advantages in being minimally invasive, can be applied in the presence of infection and allow gradual correction of the deformity. In the acute Eichenholtz stage 1, frames are often used as a static device to prevent the collapse of the tarsal bones or to treat displaced fractures and active ulcers in patients who are not suitable for total contact casting, as might be the case with obese patients or those

\begin{abstract}
${ }^{1-3}$ Department of Orthopaedics and Trauma, King's College Hospital, London, United Kingdom

Corresponding Author: Om Lahoti, Department of Orthopaedics and Trauma, King's College Hospital, London, United Kingdom, Phone: +0044 7887951880, e-mail: omlahoti@mac.com

How to cite this article: Lahoti O, Abhishetty N, Shetty S. Correction of Foot Deformities from Charcot Arthropathy with the Taylor Spatial Frame: A 7-14-year Follow-up. Strategies Trauma Limb Reconstr 2021;16(2):96-101.
\end{abstract}

Source of support: Nil

Conflict of interest: None

with amputation on the other side..$^{7-9}$ In a foot with established deformity (Eichenholtz stage 2 and 3), a circular frame is used either as a holding device after open wedge excision and the acute correction of deformity ${ }^{10}$ or to gradually correct the deformity $y^{7,8,11-13}$ without excising bone.

The aim of this study is to report the results of a strategy comprising of a minimally invasive targeted osteotomy, an acute correction of hindfoot deformity where possible and a gradual correction of midfoot deformity using a Taylor spatial frame (TSF) hexapod in patients with recurrent active ulceration due to deformity and with an active or past history of deep infection in the foot, all of which increase the risk of complications following open surgery and internal fixation. The successful outcome measures are complete healing of ulcer, clearance of infection without recurrence, a clinically plantigrade foot and the ability to use regular shoes 
or diabetic footwear. There were successful outcomes in 9 out of 10 patients. One patient did not complete the treatment for psychosocial reasons. We included this case as a failure even though the likely contributing factor was poor patient selection. The other nine patients remain ulcer- and infection-free at a minimum of 7 years and maximum of 14 years follow-up.

\section{Materials and Methods}

A range of treatments are provided at our institute for the Charcot foot deformities, including the total contact casting, open surgical correction and internal fixation and circular frame treatment (with the TSF). Our indication for the treatment with TSF is recurrent or intractable ulceration with or without active bone infection or a history of recurrent ulceration and deep infection in a deformed foot. Case selection is important for all reconstructive procedures in the diabetic neuroarthropathic population and our prerequisites were as follows:

- Confirmation of good arterial perfusion to the limb

- Being medically stable and having good cognitive function

- Being independently mobile before the Charcot process became established

Patients who did not meet these prerequisites were excluded and offered other alternative, often non-surgical, treatment.

The TSF was used in 10 patients (10 feet) to correct the Charcot arthropathy-related foot deformities between 2005 and 2013. Table 1 shows patient data and Figure 1, duration in frame. Figure 2 illustrates a case of rocker-bottom foot deformity with an ulcer (case 5 ) and forefoot $6 \times 6$ butt frame. The average age was 51 years (range 32-68). There were two female and eight male patients in this cohort. A long bone TSF module (Fig. 3, for ankle and hindfoot deformities) was used in four patients and a forefoot $6 \times 6$ butt frame in six patients with midfoot deformities.

\section{Preoperative Counselling and Preparation}

A full physical examination was conducted at the first consultation. At least two outpatient consultations were arranged to ensure that patients understood the need for a frame treatment clearly and that they were capable of compliance and had a good family and social support. A range of available treatment modalities were also discussed, including non-frame treatment options. The patients were also shown a model of a frame and offered an opportunity to meet other patients undergoing such treatment prior to consenting to the surgery. A close working relationship was established with diabetic physicians, podiatrists, specialist frame nurses and physiotherapists.

Weight-bearing anteroposterior (AP) and lateral (LAT) radiographs of the foot and ankle with hindfoot views were obtained routinely. Our experience has shown advanced imaging modalities, such as magnetic resonance imaging and nuclear medicine scans, are not helpful in decision-making because the absence of infection was not a prerequisite for frame treatment. In fact, the frame treatment is the preferred option in the presence of infection. The apex of the deformity was identified on the AP and LAT foot X-rays according to deformity analysis planning methods ${ }^{14}$ and was correlated with the clinical deformity.

\section{Operative Technique}

Surgery was performed under general anaesthesia or spinal block. The patient was positioned supine with a sandbag under the ipsilateral buttock. Appropriate antibiotics were given at induction of anaesthesia unless bone sampling was planned for suspected osteomyelitis. Deformity correction was through an osteotomy in all cases. The osteotomy was planned at the apex of the deformity or as close to it as possible, depending on the location of the ulcer, any previous surgery and the condition of soft tissues. The osteotomy was often either through cuboid and navicular or through the cuboid and cuneiform bones. Osteotomies through the bases of the metatarsals were avoided because of the potential risk to neurovascular structures and the interosseous muscles in the intermetatarsal spaces. Midfoot osteotomies were made through two small incisions, one on the lateral and another on the medial border of the foot. The path of the osteotomy was marked with two K-wires which helped to constrain the osteotomes or Gigli saw (Fig. 4A). Laminar spreaders were used from the lateral and medial wounds to confirm that the osteotomy was complete and was able to move freely; this was particularly important if the osteotomes were used to perform an osteotomy. One advantage of using a Gigli saw is that the technique itself confirms that the osteotomy is complete. In hindfoot deformity cases, the correction was achieved through a calcaneal osteotomy. A forefoot $6 \times 6$ butt (Fig. 4B) configuration was used for the midfoot correction, using distal reference and the rotatory frame angle set at $180^{\circ}$, as recommended. A hindfoot $2 / 3$ rd ring was fixed to the calcaneum (open section facing upwards and proximally) with a combination of Schanz screws and olive wires. A forefoot 2/3rd ring (open section facing plantarwards) was fixed using two olive

Table 1: Details of 10 patients

\begin{tabular}{lclll}
\hline Patient & Age/sex & \multicolumn{1}{c}{ Deformity/brodsky type } & Eichenholtz stage & TSF module \\
\hline $1 \mathrm{HP}$ & $\mathrm{M} / 62$ & Varus ankle (3A) & 2 & Long bone \\
$2 \mathrm{GA}$ & $\mathrm{M} / 59$ & Midfoot cavovarus (1) & 3 & Forefoot butt $6 \times 6$ \\
$3 \mathrm{CC}$ & $\mathrm{M} / 40$ & Midfoot cavovarus (1) & 3 & Forefoot butt $6 \times 6$ \\
$4 \mathrm{MD}$ & $\mathrm{M} / 58$ & Rocker bottom (1) & 3 & Forefoot butt $6 \times 6$ \\
$5 \mathrm{TT}$ (Fig. 2) & $\mathrm{F} / 41$ & Rocker bottom (1) & 3 & Forefoot butt $6 \times 6$ \\
$6 \mathrm{PD}$ & $\mathrm{M} / 48$ & Varus deformity (3A) & 3 & Long bone \\
$7 \mathrm{HH}$ & $\mathrm{F} / 36$ & Rocker bottom (1) & 3 & Forefoot butt $6 \times 6$ \\
$8 \mathrm{CA}$ & $\mathrm{M} / 63$ & Calcaneovalgus (3A) & 2 & Long bone \\
9 PS (Fig. 3) & $\mathrm{M} / 48$ & Hindfoot varus, collapsed talus (4) & 3 & Long bone and calcaneal osteotomy \\
10 AR & $\mathrm{M} / 68$ & Rocker bottom (1) & 3 & Forefoot butt $6 \times 6$ \\
\hline
\end{tabular}


wires through the metatarsals - a medial olive wire capturing the 1st and 2nd, with or without the 3rd metatarsal and a lateral olive wire capturing the 5th and 4th, with or without the $3 \mathrm{rd}$ metatarsals. Stirrup wires (crossed olive wires entering at the distal face of the osteotomy in Fig. 4C) were extended distally and fixed to the distal $2 / 3$ rd ring without tensioning them. A similar arrangement was used on the proximal side of the osteotomy in severe deformities. These components enabled the distraction to occur at the osteotomy site mainly and not be dissipated across the joints during the gradual correction. In hindfoot and ankle

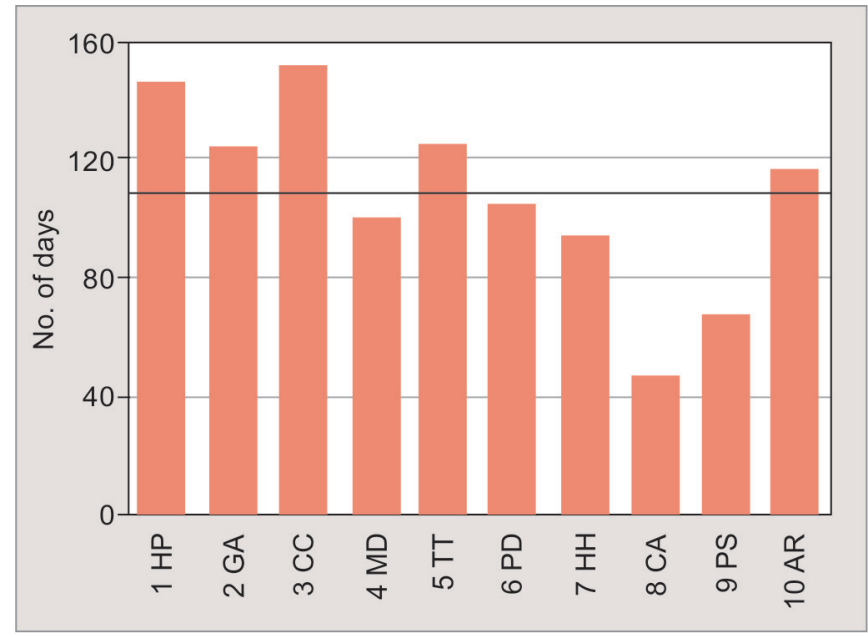

Fig. 1: Frame duration in days and average of 115 days. X-axis patient details are in Table 1. Eight CA had the frame for 47 days and treatment had to be abandoned as the patient was not coping with the frame treatment deformities (cases 1, 6, 8 and 9 in Table 1), the varus or valgus of the heel was corrected acutely through a calcaneal osteotomy but the equinus deformity through the ankle gradually by using a long bone module. With the forefoot butt construct, the hindfoot $2 / 3$ rd ring was applied first by placing it as far back as possible to create room for the short and medium struts between the forefoot and hindfoot rings. The forefoot $2 / 3 \mathrm{rd}$ ring was fixed using opposing olive wires across the metatarsal neck or heads, and the stirrup wires were brought to this ring as far forward as possible to create good space between the rings. After achieving good fixation of the two rings, about $5^{\circ}$ to $10^{\circ}$ of the deformity was corrected acutely by pulling on them. This step further increased the room between the two rings to accommodate short to medium struts. This step also reduced the overall time in the frame by acutely correcting a part of the deformity without incurring a delay in bone healing. Care was taken not to correct more than $5^{\circ}-10^{\circ}$ to avoid neurovascular compromise. Frame mounting parameters were obtained using an image intensifier with threaded rods as markers. ${ }^{15}$ Pressure dressings were applied to the osteotomy wounds and pin sites, and the leg was elevated for 12-24 hours.

Gradual correction commenced between 7 and 10 days later. Postoperative monitoring included wound checks, pin-site status and strut checks at weekly intervals. Once the foot deformity was corrected, $\mathrm{X}$-rays focussed on the osteotomy site were obtained to assess the healing. The stability at the osteotomy was also assessed by conducting a stress examination after disconnecting all the struts. If the osteotomy site was found to be stable, the frame with wires and half-pins were removed in the clinic without anaesthesia. Full weight-bearing in an Aircast boot was permitted thereafter for up to 6 months before moving on to regular shoes. Patients were followed up at 3, 6 and 12 months and then annually. Any recurrence of deformity and ulceration was noted.
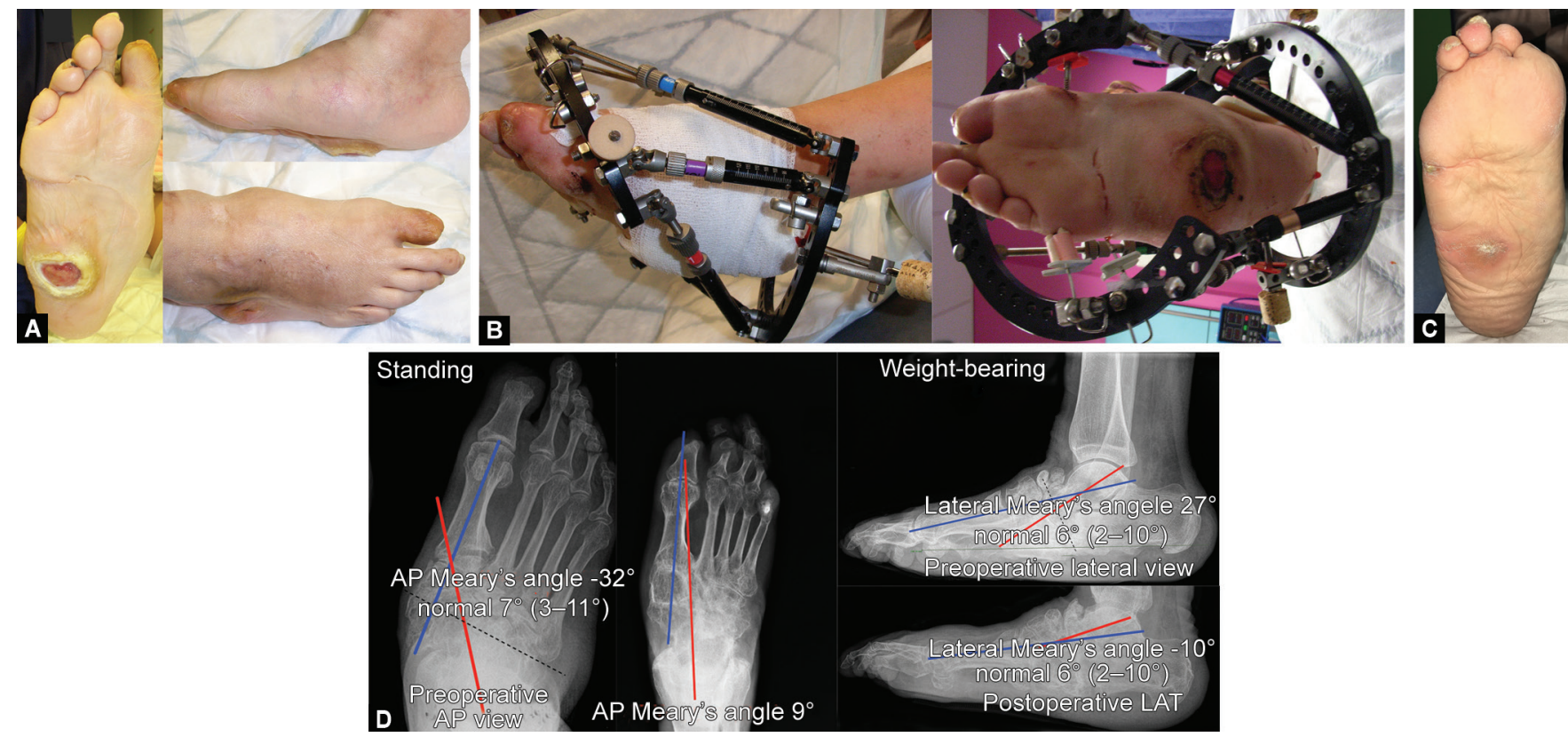

Figs 2 A to D: (A) Case 5 TT. Plantar ulcer over 3 years. Lost right big toe tip due to infection. Mild rocker bottom deformity; (B) Forefoot $6 \times 6$ butt frame; (C) Postoperative clinical 10-year follow-up. Ulcer healed and never recurred even when patient had another episode of foot infection in the little toe and it was amputated at another institution; (D) Pre- and postoperativ X-rays (interrupted black line-level of osteotomy) 


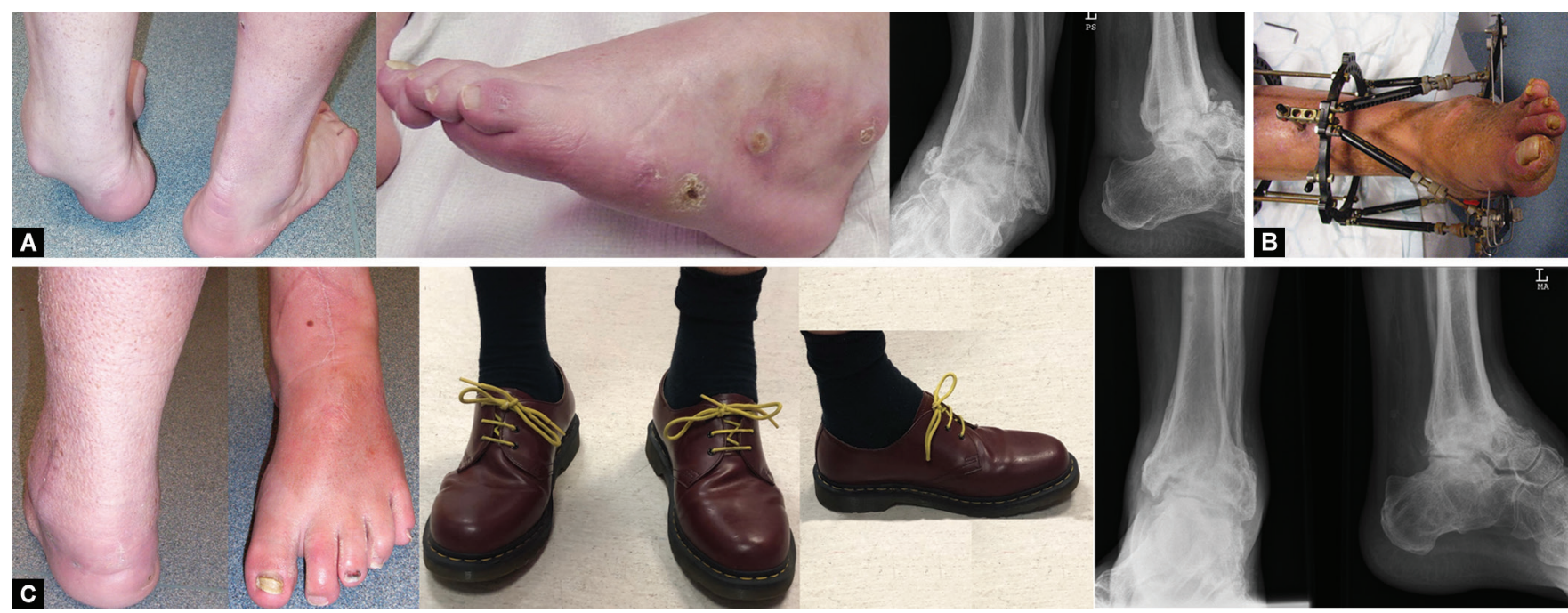

Figs 3A to C: (A) Hindfoot varus deformity and recurrent ulceration from total contact casting and bracing. Preoperative X-rays show destruction of talus and varus deformity. Managed with calcaneal osteotomy and lateral shift and gradual correction of ankle varus with TSF; (B) Long bone TSF module. Foot frame and two rings tibial block. Gradual correction of varus through collapsed talus; (C) Postcorrection clinical picture and X-rays. Hindfoot neutral and plantigrade foot. Normal off the shelf shoes. Post of X-ray shows good correction of varus and spontaneous fusion of ankle
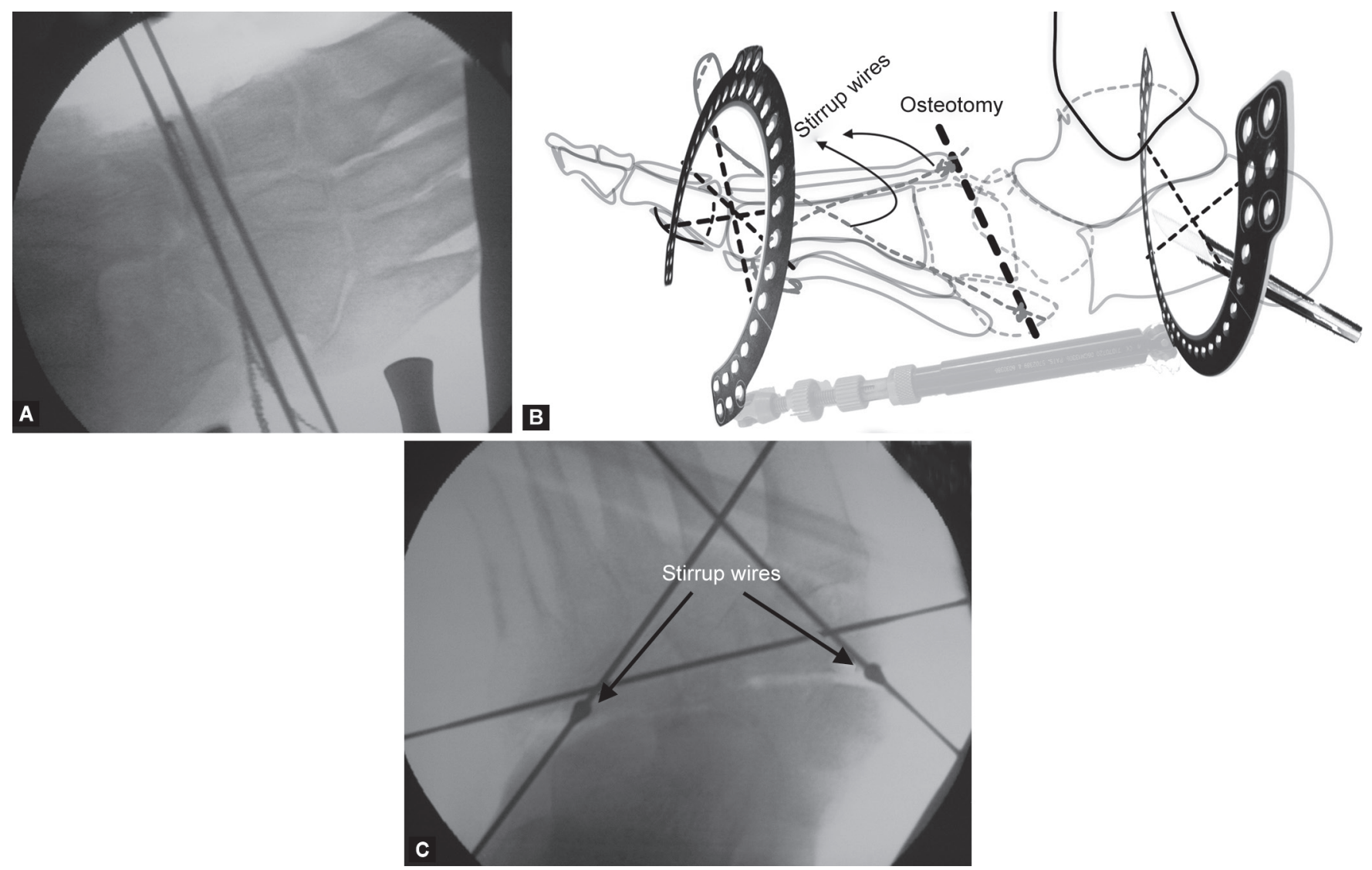

Figs 4A to C: (A) Technique of midfoot osteotomy; (B) Forefoot butt $6 \times 6$ frame configuration; (C) Olive stirrup wires

\section{Results}

Pragmatic criteria as suggested by Pinzur ${ }^{10}$ were adopted as there are no specific outcome scores or measures for Charcot neuroarthropathy-related foot deformities. A foot and ankle that was plantigrade, infection-free and able to fit into regular or diabetic therapeutic footwear was considered a successful outcome. This was achieved in 9 out of 10 patients (10 feet). In one patient, the frame treatment had to be abandoned before completion 
(case 8CA in Table 1) at 47 days due to noncompliance and poor social support. This was a failure of preoperative counselling rather than that of the technique. There were eight episodes of pin infection in 10 patients requiring 7 days of oral flucloxacillin each. It was noted that pin-site infection was less common in stable Eichenholtz stage 3 patients. Building a stable frame construct (with at least three fixation points on each ring and a good spread between the pins and screws), good preoperative counselling and close follow-up limited issues of pin breakage to just two episodes of one each in two patients. This problem was managed by removing the wire or screw in the outpatient department without adversely affecting the frame stability. None of the patients needed to return to the operating room for either pin-site infection or wire breakage. The duration of frame fixation ranged from 68 to 152 days with the average of 114 days in the nine patients who completed treatment. The one patient whose treatment was abandoned had the frame in situ for 47 days (Fig. 1). Internal fixation was used in only one case at the time of frame removal at 68 days because the patient had a confirmed pregnancy within a week of starting treatment and suffered from hyperemesis. The deformity was corrected within the first 4 weeks for most patients. The frame was then left in situ for a further 10 to 12 weeks before removal according to the protocol as in Sect. 2.1.

For the rocker-bottom foot deformities, the average preoperative AP and LAT Meary's angles were $25^{\circ}$ (Normal $3^{\circ}-11^{\circ}$ ) and $18^{\circ}$ (Normal $2^{\circ}-10^{\circ}$ ), respectively, with an improvement to $10^{\circ}$ and $8^{\circ}$, respectively. Radiographic fusion was seen on follow-up $\mathrm{X}$-rays within 1 year in all midfoot osteotomies. It was noted that the osteotomy site was stable even when the immediate postframe removal X-ray showed only patchy fusion. All osteotomies healed with full bony consolidation within 1 year of surgery. Follow-up ranged from a minimum of 7 years to a maximum of 14 years; a plantigrade ankle, with ulcer- and infection-free feet was found in 9 out of 10 patients. The correction of deformity was essential for the healing of ulcers in the long-term with the nine patients able to use regular or diabetic footwear (Fig. 3C).

\section{Discussion}

Surgical treatment of the complex foot and ankle deformities from Charcot neuroarthropathy is now established. ${ }^{16-18}$ Although better surgical techniques and modern implants have reduced the risks of surgery generally, patients with large deformities, recurrent ulceration with active or indolent osteomyelitis, a high BMI, poor bone and soft tissue quality and those who have failed standard surgical treatment pose a particular challenge. ${ }^{19}$ These cases are at risk of developing complications from extensive open surgery. The llizarov technique of using a fine-wire circular frame is becoming popular in such clinical scenarios because it is minimally invasive, provides excellent mechanical stability, is easy to adapt to a particular deformity and is ideal for cases with active infection. ${ }^{7,8,10,12,20}$ Circular frames have been used as stabilising devices (the so-called neutral frame mode) with or without internal fixation of the corrected foot ${ }^{10,20-22}$ or in dynamic mode with hinges to correct the deformities gradually. ${ }^{23,24}$ With the introduction of computer-aided technology in all spheres of orthopaedic surgery, the llizarov frame technique has also evolved with the introduction of hexapods. The TSF is a versatile hexapod and has long bone and foot modules that can correct a range of foot and ankle deformities. ${ }^{25-27}$ The TSF is also showing promising results in the area of diabetic foot deformities. ${ }^{11,13}$
Lamm et al. ${ }^{11}$ reported results of a two-stage percutaneous approach to Charcot diabetic foot deformities in 11 feet (eight patients) at 6 to 36 months using the TSF. They used a forefoot $6 \times 6$ butt frame with one or two rings distal tibial blocks, a drop-down $\mathrm{U}$ ring and a full forefoot ring. The forefoot deformity was corrected gradually with or without osteotomy. Internal fixation was then used with long screws to consolidate the gains and remove the frame early. They reported good correction of the foot deformities and no recurrence of ulcers. Roukis and Zgonis ${ }^{13}$ have described different frame configurations but the clinical results were not included.

This study reports on the outcome of percutaneous osteotomy and gradual correction of midfoot deformities and acute correction of hindfoot deformities using the TSF. A good outcome was achieved in 9 out of 10 patients. No ulcers recurred at 7-14 years follow-up. One failure occurred and was due to premature termination of treatment because of non-compliance. This case emphasises the importance of careful patient selection. Our frame construct for midfoot deformities is different from that used by Lamm et al. ${ }^{11}$ in that we used two $2 / 3 \mathrm{rd}$ rings in the foot and left the ankle-free for physiotherapy. The frame configuration in both series was not conducive to weight-bearing. Lamm et al. used internal fixation at the end of the frame correction in all their cases whereas internal fixation was used in only one case after the correction of midfoot deformities in this series. A good improvement of the midfoot deformities as evidenced from the Meary's angles from the AP and LAT radiographs (Fig. 2D) was noted. Osseous union was seen across all midfoot and calcaneal osteotomies, which helped to prevent the recurrence of deformity, even without internal fixation. In hindfoot deformities (equinovarus and calcaneovalgus), the varus or valgus component was corrected acutely through a calcaneal osteotomy whereas the ankle equinus was corrected gradually using a long bone module of the TSF. This combination reduced the complexity of the frame and also reduced the time in the frame.

Pin-site infections are an inherent obstacle in the circular frame treatment, more so in diabetic patients. Pin breakage is also reported commonly in diabetic patients due to diminished pain in neuropathic feet and uncontrolled weight-bearing. However, a meticulous wiring technique, the use of hydroxyapatite-coated screws, careful patient selection, diligent pin-site hygiene, the early use of oral antibiotics, as soon as the infection is suspected, and regular follow-up, either face-to-face in a clinic or remotely, have helped us to keep the complications to a minimum.

Treatment with a circular frame is complex and needs a team approach. Frequent follow-ups during correction consume considerable healthcare resources. However, these issues should be weighed against the risk of limb loss in such a complex cohort of patients. Fluctuations in medical comorbidities, such as cardiac, renal and glycaemic control, have the potential to adversely affect the frame treatment and outcomes. Excellent support from physicians cannot be overemphasised. In our experience, a stable deformity cohort (Eichenholtz 3) with better control of diabetes and comorbidities does well following surgical treatment, including frame treatment.

The strength of this study is that it reports medium-term results (minimum 7 and maximum 14 years) in complex foot deformities after Charcot neuroarthropathy using the very versatile TSF modules. We recognise the shortcomings of this study. The results apply to a carefully selected group of patients who fulfilled certain prerequisites, and the surgery was performed at a tertiary 
limb reconstruction referral centre with good team support and experience with frame treatment. This was not a uniform group of deformities. We used a pragmatic outcome measure which applies to all Charcot-related foot deformities rather than deformity-specific outcomes but the technique and results described will add to the range of options available for the treatment of difficult foot deformities with recurrent ulcerations.

\section{Conclusion}

Treatment based on the TSF is a good option in carefully selected complex foot and ankle deformities from Charcot neuroarthropathy. Ulcer-free and infection-free plantigrade feet that were able to don the standard diabetic footwear were obtained after treatment in 9 out of 10 feet, with these good outcomes maintained at the 7- to 14-year follow-up review.

\section{Acknowledgements}

We are very grateful to Prof Edmonds and his diabetic foot team for their enormous help in supporting the medical care of these patients.

\section{Additional Information}

The Taylor Spatial Frame.

Manufactured by: Smith and Nephew, 1450 Brooks Road Memphis, Tennessee 38116, USA

\section{References}

1. McKenzie P. Diabetes footcare project 1: pathway development. North West Coast Strategic Clinical Networks, 2017.

2. Armstrong DG, Wrobel J, Robbins JM. Guest editorial: are diabetesrelated wounds and amputations worse than cancer? Int Wound J 2007;4(4):286-287. DOI: 10.1111/j.1742-481X.2007.00392.x.

3. Paisey RB, Abbott A, Paisey CF, et al. Diabetic foot ulcer incidence and survival with improved diabetic foot services: an 18-year study. Diabet Med 2019;36(11):1424-1430. DOI: 10.1111/dme.14045.

4. La Fontaine J, Lavery L, Jude E. Current concepts of Charcot foot in diabetic patients. Foot 2016;26:7-14. DOI: 10.1016/j.foot.2015.11.001.

5. Louwerens JWK. Reconstruction of the medial column of the foot using plate fixation. Fuß Sprunggelenk 2016;14(4):212-218. DOI: 10.1016/j.fuspru.2016.08.009.

6. Richman J, Cota A, Weinfeld S. Intramedullary nailing and external ring fixation for tibiotalocalcaneal arthrodesis in charcot arthropathy. Foot Ankle Int 2017;38(2):149-152. DOI: $10.1177 / 1071100716671884$.

7. Yousry AH, Abdalhady AM. Management of diabetic neuropathic ankle arthropathy by arthrodesis using an llizarov frame. Acta Orthop Belg 2010;76(6):821-826. Available at: https://pubmed.ncbi.nlm.nih. gov/21302582/

8. El-Gafary KA, Mostafa KM, Al-Adly WY. The management of Charcot joint disease affecting the ankle and foot by arthrodesis controlled by an Ilizarov frame: early results. J Bone Joint Surg Br 2009;91(10):13221325. DOI: 10.1302/0301-620X.91B10.22431.

9. Kimawi AA, Snyder RJ, Cala MA, et al. When traditional offloading is not an option, could an external fixator be a solution? A case report.
Wounds 2017;29(2):46-50. Available at: https://pubmed.ncbi.nlm. nih.gov/28272013/

10. Pinzur MS. Static ring fixation for nonplantigrade charcot midfoot deformity. Oper Tech Orthop 2008;18(4):287-292. DOI 10.1053/j. oto.2009.01.006

11. Lamm BM, Gottlieb HD, Paley D. A two-stage percutaneous approach to charcot diabetic foot reconstruction. J Foot Ankle Surg 2010;49(6):517-522. DOI: 10.1053/j.jfas.2010.07.014.

12. Ramanujam $\mathrm{CL}$, Zgonis T. An overview of internal and external fixation methods for the diabetic charcot foot and ankle. Clin Podiatr Med Surg 2017;34(1):25-31. DOI: 10.1016/j.cpm.2016.07.004.

13. Roukis TS, Zgonis T. The management of acute charcot fracturedislocations with the Taylor spatial external fixation system. Clin Podiatr Med Surg 2006;23(2):467-483. DOI: 10.1016/j. cpm.2006.01.008.

14. Shawn C. Standard MJEH, Conway JD, et al. The art of limb alignment: chapter 11. In: Foot and ankle axis planning: frontal, sagittal, and transverse planes, 7th ed. Baltimore: Rubin Institute for Advanced Orthopedics, Sinai Hospital of Baltimore, 2018. p. 221.

15. Belczyk RJ, Wukich DK. Correction of rockerbottom deformity using the butt frame. Oper Tech Orthop 2006;16(1):23-31. DOI 10.1053/j. oto.2006.02.005.

16. Frykberg RG, Wukich DK, Kavarthapu V, et al. Surgery for the diabetic foot: a key component of care. Diabetes Metab Res Rev 2020;36(Suppl 1):e3251. DOI: 10.1002/dmrr.3251.

17. Zgonis T, Stapleton JJ, Jeffries LC, et al. Surgical treatment of charcot neuroarthropathy. AORN J 2008;87(5):971-990. DOI: 10.1016/j. aorn.2008.03.002.

18. Pinzur MS, Schiff AP. Deformity and clinical outcomes following operative correction of charcot foot: a new classification with implications for treatment. Foot Ankle Int 2018;39(3):265-270. DOI: $10.1177 / 1071100717742371$.

19. Pinzur MS, Gil J, Belmares J. Treatment of osteomyelitis in Charcot foot with single-stage resection of infection, correction of deformity, and maintenance with ring fixation. Foot Ankle Int 2012;33(12):1069-1074. DOI: 10.3113/FAI.2012.1069.

20. Farber DC, Juliano PJ, Cavanagh PR, et al. Single stage correction with external fixation of the ulcerated foot in individuals with Charcot neuroarthropathy. Foot Ankle Int 2002;23(2):130-134. DOI: $10.1177 / 107110070202300209$.

21. Grant WP, Garcia-Lavin SE, Sabo RT, et al. A retrospective analysis of 50 consecutive charcot diabetic salvage reconstructions. J Foot Ankle Surg 2009;48(1):30-38. DOI: 10.1053/j.jfas.2008.10.004.

22. Stapleton JJ, Zgonis T. Surgical reconstruction of the diabetic charcot foot: internal, external or combined fixation? Clin Podiatr Med Surg 2012;29(3):425-433. DOI: 10.1016/j.cpm.2012.04.003.

23. Cooper PS. Application of external fixators for management of charcot deformities of the foot and ankle. Semin Vasc Surg 2003;16(1):67-78. DOI: $10.1053 /$ svas.2003.50007.

24. Paley D. Principles of deformity correction, 1st ed. New York: Springer, 2002.

25. Floerkemeier $\mathrm{T}$, Stukenborg-Colsman $\mathrm{C}$, Windhagen $\mathrm{H}$, et al. Correction of severe foot deformities using the Taylor spatial frame. Foot Ankle Int 2011;32(2):176-182. DOI: 10.3113/FAI.2011.0176.

26. Waizy $\mathrm{H}$, Windhagen $\mathrm{H}$, Stukenborg-Colsman $\mathrm{C}$, et al. Taylor spatial frame in severe foot deformities using double osteotomy: technical approach and primary results. Int Orthop 2011;35(10):1489-1495. DOI: 10.1007/s00264-011-1269-8.

27. Eidelman M, Katzman A. Treatment of arthrogrypotic foot deformities with the Taylor spatial frame. J Pediatr Orthop 2011;31(4):429-434. DOI: 10.1097/BPO.0b013e3182172392. 